\section{Window of opportunity}

The transcription factor hunchback $(\mathrm{Hb})$ specifies early-bornneuron identity within embryonic neural progenitor (neuroblast) lineages in Drosophila melanogaster. Transcription of the $h b$ gene is active only during the first two neuroblast cell divisions. Yet, neuroblasts maintain the capacity

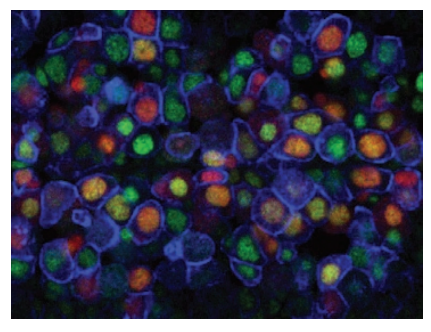

to induce early-born, so-called U1 and U2 neurons in the presence of ectopic $\mathrm{Hb}$ for another three divisions, after which they no longer respond to $\mathrm{Hb}$. Doe and colleagues have investigated the underlying mechanism that defines this competence window. They showed by immuno-DNA FISH that the $h b$ genomic locus relocates from the nuclear interior to the nuclear periphery in a near-synchronous manner in the entire neuroblast population, concurrently with the end of the competence window. The nuclear periphery is known as a highly repressive domain, and, indeed, depletion of the nuclear-lamina component lamin reduced $h b$ gene silencing. In addition, decreased lamin levels reduced the extent of $h b$ repositioning and extended the neuroblast competence window. Movement of $h b$ to the nuclear periphery correlated with downregulation of distal antenna (Dan), a nuclear protein of unknown function and mechanism, and prolonging Dan expression blocked $h b$ gene movement and extended neuroblast competence. The authors propose a mechanism whereby $h b$ is transcriptionally active in the nuclear interior for two progenitor divisions. However, $h b$ remains accessible in the nuclear interior and retains the capacity to respond to ectopic $\mathrm{Hb}$ for three more divisions until Dan is downregulated, which results in the movement of the $h b$ locus to the nuclear lamina. At this point, ectopic $\mathrm{Hb}$ can no longer induce its transcription, and the progenitor competence window is closed. (Cell 152, 97-108, 2013)

AH

\section{A IncRNA for the active $X$}

Long noncoding RNAs (lncRNAs) can modulate gene expression in various ways, including by gene silencing. In fact, one of the bestcharacterized lncRNAs is XIST, a lncRNA with a central role in $\mathrm{X}$-chromosome inactivation in placental mammals. XIST is transcribed from and coats the inactive $\mathrm{X}$ chromosome, and, together with other lncRNAs, it promotes the recruitment of histone-modifying complexes to initiate and maintain X-chromosome silencing. Now Rougelle and colleagues have identified a new lncRNA, called XACT (for X-active coating transcript), that coats the active $\mathrm{X}$ chromosome. XACT is expressed from the active $\mathrm{X}$ chromosome in human embryonic stem cells but undergoes silencing upon differentiation, and its expression could not be detected in fibroblasts or in a number of examined tissues. In cells where XIST expression is lost, XACT is expressed from both $\mathrm{X}$ chromosomes. XACT does not seem to be conserved in mice, as indicated by RNA FISH analyses of mouse embryonic stem cells. The authors propose that XACT is involved in controlling the initiation of $\mathrm{X}$-chromosome inactivation in the human embryo, a process that occurs with different kinetics compared to those of the mouse embryo. Future work will elucidate the precise role of XACT, but its identification as a lncRNA that coats active chromatin expands the already diverse functions that lncRNAs can exert in regulating gene expression. (Nat. Genet. doi:10.1038/ng.2530, published online 20 January 2013)

IC

Written by Inês Chen, Arianne Heinrichs, Stéphane Larochelle \& Beth Moorefield

\section{STARR-seq enterprise}

Transcriptional enhancers are key determinants of cellular differentiation and development, but the fact that they function in a position-independent and cell type-specific manner often complicates their identification. Now, Stark and colleagues describe a reporter-based strategy that takes full advantage of the position-independent nature of enhancer function to generate a quantitative genomic map of enhancer activity in Drosophila cells. In their STARR-seq (self-transcribing active regulatory region sequencing) approach, candidate sequences are cloned downstream of a minimal promoter, such that enhancers will stimulate their own transcription. Enhancer strength is thus reflected by the relative abundance of enhancer transcripts. STARR-seq analysis of a fly genomic DNA library assayed in S2 cells identified $\sim 5,500$ enriched sequences exhibiting a range of enhancer strengths. Validation of representative subsets with luciferase reporters confirmed the predicted strong and weak enhancer activity of the STARR-seq isolates, which mapped to both intronic and intergenic regions. DNase accessibility assays to assess the chromatin state of these enhancers at their endogenous genomic locations revealed that the majority of strong enhancers and many weak enhancers were in an 'open' configuration and had the H3K27ac mark characteristic of active elements. The remaining strong enhancers that displayed a 'closed' configuration were associated with a repressive $\mathrm{H} 3 \mathrm{~K} 27 \mathrm{me} 3$ signature, which suggests that they are actively repressed in S2 cells. Comparative STARR-seq analysis of Drosophila ovarian somatic cells revealed cell type-specific differences in enhancer strength that correlated well with differential expression of genes flanking the endogenous enhancer loci. Thus, STARR-seq's ectopic enhancer-activity assays provide a complementary approach to current chromatin-based methods of identifying genomic enhancers. (Science doi:10.1126/science.1232542, published online 17 January 2013)

$B M$

\section{Directing DNA demethylation}

The presence of 5 -methylcytosine $(5 \mathrm{mC})$ at gene promoters suppresses transcription, but the molecular mechanisms leading to derepression through active, site-specific DNA demethylation are poorly understood. The DNA damage-associated protein Gadd45a was previously suggested to participate in selective demethylation at the promoters of target genes through recruitment of the DNA repair machinery, but how it was targeted to specific sites remained unknown. Schäfer et al. now implicate the tumor suppressor Ing1-which contains a PHD finger domain that specifically binds $\mathrm{H} 3 \mathrm{~K} 4 \mathrm{me} 3$ - in DNA demethylation by recruiting Gadd45a to $\mathrm{H} 3 \mathrm{~K} 4 \mathrm{me} 3$ sites. Ing1 and Gadd45a were found to interact, and cotransfection of Ing 1 greatly stimulated the ability of Gadd45a to demethylate and reactivate the promoters of a reporter gene and the endogenous Mageb2 target gene. The stimulatory activity of Ing 1 was dependent on an intact PHD domain, and reducing the level of $\mathrm{H} 3 \mathrm{~K} 4 \mathrm{me} 3$ by knocking down Wrd5-a component of the MLL methyl-transferase complex - prevented the recruitment of Gadd45a and Ing1, increased $5 \mathrm{mC}$ levels at the promoter and impaired the induction of Mageb2 transcription. Finally, genome-wide changes in gene expression induced by the transfection of Gadd45a and Ing1 depended on the presence of $\mathrm{H} 3 \mathrm{~K} 4 \mathrm{me} 3$ and correlated with reduced levels of DNA methylation at the promoters of target genes. These observations demonstrate that histone methylation can dynamically direct site-specific DNA demethylation and promote gene expression by recruiting specific histone readers, which in turn serve as adaptors for the demethylation machinery. (Genes Dev. 27, 261-273, 2013) 